\title{
Editorial
}

\section{Is There A Place for Systemic Chemotherapy and Targeted Agents in Metastatic Small Intestine Neuroendocrine Tumors?}

\author{
Kosmas Daskalakis ${ }^{1}$ and Apostolos V Tsolakis ${ }^{2 *}$ \\ ${ }^{1}$ Institution of Surgical Sciences, Uppsala University, Uppsala, Sweden \\ ${ }^{2}$ Department of Gastrointestinal Endoscopy, Karolinska University Hospital Huddinge, Stockholm, Sweden \\ *Corresponding author: Apostolos V. Tsolakis, Department of Gastrointestinal Endoscopy, Karolinska \\ University Hospital Huddinge, Stockholm, Sweden, Email: apobtsol@hotmail.com ; apostolos.tsolakis@ki.se
}

Received: 13 June, 2018; Accepted: 09 August, 2018; Published: 30 August, 2018

Neuroendocrine tumors (NETs) demonstrate a widely variable clinical behavior but in general they have an indolent clinical course. Small intestine NETs (SI-NETs) of Grades 1 and 2 have rather limited response rates to systemic chemotherapy of about $10 \%-30 \%$ [1-2]. Reports in the literature describe the low activity of chemotherapeutic drugs such as $5-\mathrm{FU} /$ capecitabine given in combination to either oxaliplatin or dacarbazine/epirubicin or temozolomide [3-6]. Nevertheless, somatostatin analogs (SSAs) can control hormonal symptoms in the majority of the patients. Furthermore, SSAs show antiproliferative action. However, it is known that occasional well-differentiated SI-NETs may show a discrepancy between the proliferative index of the primary and the metastasis, necessitating the prompt initiation of systemic treatment [7].

In the past, the medical choices for treating advanced and disseminated low-proliferative SI-NETs have not been truly effective. The general consensus was that the initial treatment of metastatic SI-NETs with cytotoxic drugs is of limited benefit $[1,8]$. Generally, there have only been few placebo-controlled randomized studies so far using the Response Evaluation Criteria in Solid Tumors (RECIST) [9], with the majority of studies in the literature being small Phase II trials. Thus, as high-level evidence is unfortunately unavailable to date, there are no guidelines in favor of the use of one specific chemotherapeutic drug versus another.

Even though there may be no general advantage to treating SINETs with traditional chemotherapy, there may be some value for well-differentiated, pre-treated NET patients demonstrating disease progression. Generally, in patients with low proliferative SI-NETs, non-cytotoxic drugs may be preferred earlier in the disease course, keeping chemotherapy as a salvage option. Grade
2 SI-NETs could be potentially considered in a multidisciplinary setting in order to recognize those selected patients for whom chemotherapy might be beneficial when other modalities fail. Validation of predictive factors and development of predictive biomarkers is, of course, mandatory in order to facilitate individualized chemotherapeutic choices. However, to date we mainly have no such markers and prospective studies are yet to be performed to examine the usefulness of Ki-67 in SI-NETs. Indeed, we still do not have a clear understanding of the possible role of chemotherapeutic drugs concerning metastatic SI-NETs.

NET biology has been clarified to some extent in recent years, paving the way for the creation of novel therapies. Aberrant activation of the mammalian target of rapamycin (mTOR) pathway, as well as enhanced angiogenesis might be essential in NET pathogenesis and progression [10]. The mTOR, a prime protein kinase downstream to the phosphoinositide 3-kinase/Akt signaling pathway, is a crucial intracellular mediator involved in various functions, such as apoptosis, proliferation, differentiation, angiogenesis and tumorigenesis. Changes within mTOR itself and of mTOR-related kinases in this pathway have been recognized in NETs, making the mTOR pathway an appealing target in NET patients. Additionally, the most important trait of NETs is a paradoxically high vascularization in neoplasms with low proliferative index and a hypoxia-dependent angiogenesis drive in NETs of higher grade, which is due to the expression of pro-angiogenic factors. As a result, several anti-angiogenic compounds have been investigated in NETs, including targeting the vascular endothelial growth factor (VEGF) and its receptor (VEGFR), as well as the platelet-derived growth factor receptor (PDGFR).

The mTOR inhibitor everolimus has recently been approved as a robust treatment for disease control if other therapeutic choices 
Citation: Daskalakis K, Tsolakis AV (2018) Is There A Place for Systemic Chemotherapy and Targeted Agents in Metastatic Small Intestine Neuroendocrine Tumors? J Clin Gastroenterol Dig Disord 2018: 1-3. DOI: https://doi.org/10.29199/CGDD-101011.

are proven unsuccessful [11-12]. According to the RAD001 in Advanced Neuroendocrine Tumors Second Trial (RADIANT-2 study), everolimus plus octreotide LAR treatment appeared to offer survival advantages in patients with advanced SI-NETs and associated carcinoid syndrome [11]. Additionally, everolimus, as a single agent, has shown vigorous anti-tumor activity and is reasonably well-tolerated by patients across a broad range of neuroendocrine (non-pancreatic) tumors, as seen in the RADIANT-4 study [12]. Furthermore, sunitinib and pazopanib hydrochloride, both multiple tyrosine kinase inhibitors, are currently being evaluated separately in randomized Phase II trials, [NCT01731925 (a Study of Sunitinib Versus Placebo in Combination with Lanreotide in Patients with Progressive Advanced/ Metastatic Midgut Carcinoid Tumors, the SUNLAND trial) and NCT00454363 (Pazopanib Hydrochloride in Treating Patients with Advanced Neuroendocrine Cancer)] in progressive, advanced or metastatic SI-NETs. A recent study on the ex vivo cytotoxic activity of traditional chemotherapy and newly introduced targeted agents in SI-NETs exhibited intermediate, yet variable sensitivity, requiring systemic therapies that are individualized for each SI-NET patient [13]. Additionally, the biomarkers in current use for SI-NETs, i.e Chromogranin A and 5-HIAA, along with clinicopathological factors, are not unambiguously associated with susceptibility to chemotherapy and targeted agents, which challenges these criteria in clinical practice [13].

Finally, as well-differentiated SI-NETs are rather resistant to conventional chemotherapy, the recently demonstrated anti-tumoral activity of newly introduced targeted agents has sparked fresh optimism among researchers. Blending these newly introduced targeted agents with chemotherapy may be an attractive option, since such a combined approach might prevent the development of resistance or escape mechanisms, which is often encountered in NET patients. Taken together, chemotherapy and targeted agents may complete the armamentarium of systemic treatments in disseminated SI-NETs, as indicated for each patient. The development of markers to monitor and predict treatment response to these systemic agents may be the most important future task in the efforts towards individualized and precision medicine in the field of NETs.

\section{References}

1. Sun W, Lipsitz S, Catalano P, Mailliard JA, Haller DG, et al. (2005) Phase II/III study of doxorubicin with fluorouracil compared with streptozocin with fluorouracil or dacarbazine in the treatment of advanced carcinoid tumors: Eastern Cooperative Oncology Group Study E1281. J Clin Oncol 23: 4897-4904.
2. Faure M, Niccoli P, Autret A, Cavaglione G, Mineur L, et al. (2017) Systemic chemotherapy with FOLFOX in metastatic grade 1/2 neuroendocrine cancer. Mol Clin Oncol 6: 44-48.

3. Bajetta E, Catena L, Procopio G, De Dosso S, Bichisao E, et al. (2007) Are capecitabine and oxaliplatin (XELOX) suitable treatments for progressing low-grade and high-grade neuroendocrine tumours? Cancer Chemother Pharmacol 59: 637-642.

4. Walter T, Bruneton D, Cassier PA, Hervieu V, Pilleul F (2010) Evaluation of the combination 5-fluorouracil, dacarbazine, and epirubicin in patients with advanced well-differentiated neuroendocrine tumors. Clin Colorectal Cancer 9: 248-254.

5. Fine RL, Gulati AP, Krantz BA, Moss RA, Schreibman S, et al. (2013) Capecitabine and temozolomide (CAPTEM) for metastatic, well-differentiated neuroendocrine cancers: The Pancreas Center at Columbia University experience. Cancer Chemother Pharmacol 71: 663-670.

6. Crespo G, Jimenez-Fonseca P, Custodio A, Lopez C, Carmona-Bayonas $A$,et al. (2017) Capecitabine and temozolomide in grade 1/2 neuroendocrine tumors: a Spanish multicenter experience. Future Oncol 13: 615-624.

7. Shi C, Gonzalez RS, Zhao Z, Koyama T, Cornish TC, et al. (2015) Liver metastases of small intestine neuroendocrine tumors: Ki-67 heterogeneity and World Health Organization grade discordance with primary tumors. Am J Clin Pathol 143: 398-404.

8. Kunz PL, Reidy-Lagunes D, Anthony LB, Bertino EM, Brendtro K, et al. (2013) Consensus guidelines for the management and treatment of neuroendocrine tumors. Pancreas 42: 557-577.

9. Eisenhauer EA, Therasse P, Bogaerts J, Schwartz LH, Sargent D, et al. (2009) New response evaluation criteria in solid tumours: revised RECIST guideline (version 1.1). Eur J Cancer 45: 228-247.

10. Qian ZR, Ter-Minassian M, Chan JA, Imamura Y, Hooshmand SM, et al. (2013) Prognostic significance of MTOR pathway component expression in neuroendocrine tumors. J Clin Oncol 31: 3418-3425.

11. Pavel ME, Hainsworth JD, Baudin E, Peeters M, Horsch D, et al. (2011) Everolimus plus octreotide long-acting repeatable for the treatment of advanced neuroendocrine tumours associated with carcinoid syndrome (RADIANT-2): a randomised, placebo-controlled, phase 3 study. Lancet 378: 2005-2012.

12. Yao JC, Fazio N, Singh S, Buzzoni R, Carnaghi C, et al. (2016) Everolimus for the treatment of advanced, non-functional neuroendocrine tumours of the lung or gastrointestinal tract (RADIANT-4): a randomised, placebo-controlled, phase 3 study. Lancet 387: 968977.

13. Daskalakis K, Norlen O, Karakatsanis A, Hellman P, Larsson R, et al. (2018) Ex vivo activity of cytotoxic drugs and targeted agents in small intestinal neuroendocrine tumors. Endocr Relat Cancer 25: $471-480$. 


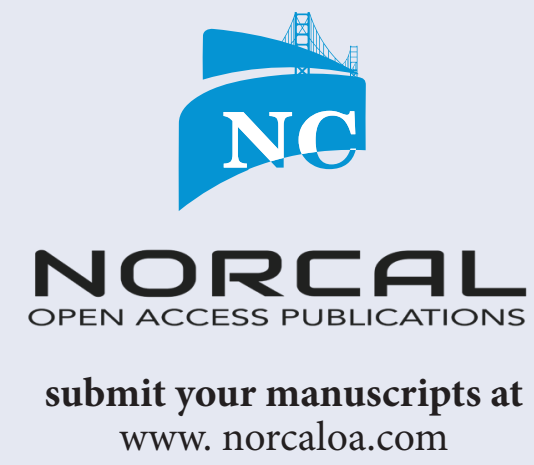

www. norcaloa.com 